\title{
Kondo effect in Complex Quantum Dots in the presence of an oscillating and fluctuating gate signal
}

\author{
M.N. Kiselev, ${ }^{1}$ K. Kikoin, ${ }^{2}$ and J. Richert ${ }^{3}$ \\ ${ }^{1}$ The Abdus Salam International Centre for Theoretical Physics, Strada Costiera 11, I-34151 Trieste, Italy \\ ${ }^{2}$ School of Physics and Astronomy, Tel-Aviv University 69978, Israel \\ ${ }^{3}$ Institut de Physique, Université de Strasbourg, 3, \\ Rue de l'Université, 67084 Strasbourg Cedex, France
}

(Dated: June 4, 2018)

\begin{abstract}
We show how the charge input signal applied to the gate electrode in a double and triple quantum dot may be converted to a pulse in the Kondo cotunneling current being a spin response of a nanodevice under a strong Coulomb blockade. The stochastic component of the input signal results in the infrared cutoff of Kondo transmission. The stochastization of the orbital component of the Kondo effect in triple quantum dots results in a noise-induced $S U(4) \rightarrow S U(2)$ quantum transition.

PACS numbers: 73.23.Hk, 72.15.Qm, 73.21.La, 73.63.-b,
\end{abstract}

\section{INTRODUCTION}

Current interest in charge-spin conversion effects is spurred by challenging prospects of spintronics. Most of the mechanisms of such a conversion are related to the interconnection between electrical and spin current due to spin-orbit interaction, $\frac{1}{\underline{p}}$ which results in spin accumulation near the sample edges. Such an accumulation in three- and two-dimensional electron gas in elemental and III-V semiconductors may result in a spin-Hall effect ${ }^{2}$ and positive magnetoresistance. $\frac{3}{3}$ It was argued also that the Rashba-type spin-orbit interaction in a quantum dot assists pure spin current by modulation of the voltages applied to the leads in a three-terminal device. $\underline{\underline{4}}$ Spin Coulomb drag effects should be mentioned in this context, which may result in spin polarization of charge current due to intrinsic friction between electrons with different spin projections induced by Coulomb scattering, $\underline{\underline{5}, \underline{6}}$

In all these propositions the possibilities of conversion of charge current into spin current were discussed. It is possible also to try to use the external electric field for the generation of spin current or another spin response. One such idea was formulated recently for light emitting diodes (LED) based on conjugated polymers,,$\frac{7}{\underline{T}}$ where dissociation of excitons in a strong enough electric field may result in the accumulation of up and down spin densities near the two ends of the LED.

In this paper we show that the charge input signal applied to the gate electrode in double (DQD) and triple quantum dots (TQD) forming closed loops (equilateral triangle) may be conversed to a pulse in Kondo cotunneling current, which is in fact the spin response of DQD under strong Coulomb blockade. The general idea of such a conversion was formulated in our previous paper ${ }^{8}$ (hereafter referred as I). Using the example of a T-shaped DQD occupied by two electrons we have shown that the charge-spin conversion is possible due to the specific dynamical symmetry of a spin multiplet consisting of two singlets and a triplet. Since the states in this multiplet are constrained by Casimir operators of the group $S O(5)$ characterizing the dynamical symmetry, $\underline{9}$ the time-dependent perturbation in the charge sector which affects only the singlet states results in a timedependent potential acting on the triplet states.

An important aspect of the problem is the interplay between the coherent (adiabatic) and stochastic components of the input signal. In paper I we concentrated mainly on the coherent signal and discussed in details the conversion of charge noise into spin response in Kondo tunneling. This response may be interpreted as dephasing and decoherence of the Kondo screening. Here we develop the general scheme, where both coherent and stochastic response of Kondo tunneling to the time-dependent gate voltage are calculated. Besides, the decoherence due to the noise component of the input signal is considered in the long relaxation limit contrary to I, where the white noise approximation was considered for the noise correlation function.

Another mechanism of charge-to-spin conversion may be realized in an equilateral triangular TQD in an external magnetic field penetrating the triangle plane. This model was introduced in Ref. 10 with the purpose of studying the interference between Kondo tunneling and Aharonov-Bohm interference (see Ref. 11 for an experimental realization). The actual dynamical symmetry of TQD in a contact with metallic electrodes is $S U(4)$ because the system possesses two spin and two orbital degrees of freedom. We show in this paper that the time dependent gate potential affects only the orbital component of multiplet, but the Kondo tunneling is sensitive to this perturbation because both orbital and spin discrete degrees of freedom are involved in Kondo screening.

\section{COHERENT AND STOCHASTIC CHARGE SIGNAL}

The subject of our calculations is the study of the transformation of the charge input signal into a Kondo response of complex quantum dots in tunnel contact with 
source and drain electrodes. We study the mechanism of activation of internal spin degrees of freedom by means of a time-dependent gate potential applied to passive electrode. Two examples of complex nano-devices will be considered. The first is an asymmetric double quantum dot (DQD) which contacts with metallic leads in a so called T-shape geometry (Fig. 17). The second is a triple quantum dot (TQD) in the form of an equilateral triangle in a three-terminal configuration, where the bias is established between the dots 2,3 and the time-dependent gate potential is applied to the dot 1 ((Fig. 10).

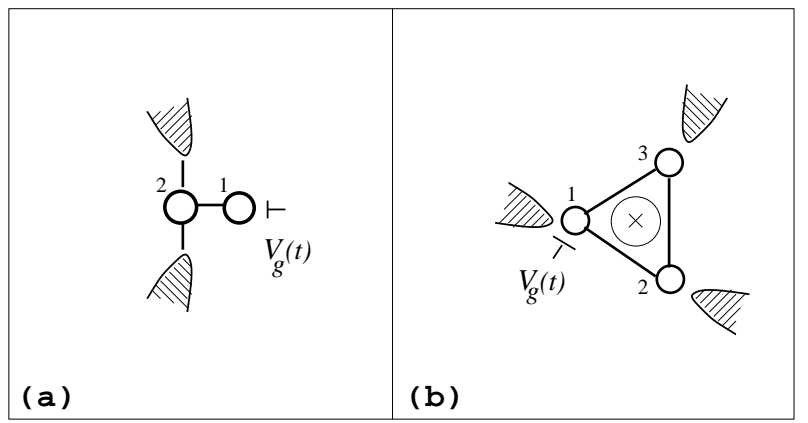

FIG. 1: (a) Double quantum dot in a T-shape two-terminal geometry (b) Triangular quantum dot in a three-terminal geometry in a magnetic field perpendicular to the plane of the triangle.

In both cases the electron tunneling is described by the Anderson Hamiltonian

$$
H=H_{\text {band }}+H_{d o t}+H_{t u n}
$$

where three terms are related to band electrons in the leads, electrons in the complex dot and tunneling coupling between two subsystems, respectively. The lead Hamiltonian has the form

$$
H_{b a n d}=\sum_{b} \varepsilon_{b k} c_{b k \sigma}^{\dagger} c_{b k \sigma}
$$

Here $b$ stands for the leads, $k, \sigma$ are the wave vector and spin projection, respectively. The leads are identical in our model, $\left(\varepsilon_{b k} \equiv \varepsilon_{k}\right)$ so it is convenient to re-expand the lead electron states $c_{b k \sigma}^{\dagger}$ in terms of irreducible basis states $c_{\beta k \sigma}^{\dagger}$ of the corresponding point symmetry groups (mirror symmetry for DQD and triangular symmetry for TQD).

The Hamiltonian of the dot is written as

$$
H_{d o t}=\sum_{j} H_{j}^{0}+\sum_{j j^{\prime}}^{j \neq j^{\prime}} H_{j j^{\prime}}^{0}+H_{1}(t)
$$

The potential wells in a complex QD are enumerated by the index $j$. Here

$$
H_{j}^{0}=\varepsilon_{j} n_{j}+Q_{j} n_{j}^{2}
$$

describes electron states in the potential well $j$ under the Coulomb blockade $Q_{j}$, and $n_{j}$ are the occupation number operators. The second term

$$
H_{j j^{\prime}}^{0}=V \sum_{\sigma} d_{j \sigma}^{\dagger} d_{j^{\prime} \sigma}
$$

stands for the interdot tunneling. By convention $j=1$ is reserved for the well coupled to the gate. This coupling is described by the last term in (2.3),

$$
H_{1}(t)=\left[V_{g}(0)+v_{g}(t)\right] n_{1},
$$

where the gate voltage contains both a static component $V_{g}(0)$ and a time-dependent perturbation $v_{g}(t)$.

The tunneling term in the Hamiltonian (2.1) has the form

$$
H_{t u n}=W \sum_{j \beta} \sum_{k \sigma}\left(c_{\beta k \sigma}^{\dagger} d_{j \sigma}+H . c .\right)
$$

The values of $j$ and $\beta$ are determined by the geometry of the complex QD (see below).

We consider a general situation, where the gate potential applied to a multi-valley complex QD contains both coherent and stochastic components

$$
v_{g}(t)=\tilde{v}_{g}(t)+\delta v_{g}(t)
$$

The $\tilde{v}_{g}(t)$ is the coherent (deterministic) contribution and $\delta v_{g}(t)$ is the stochastic noise component which is defined by its moments

$$
\begin{aligned}
& \overline{\delta v_{g}(t)}=0 \\
& \overline{\delta v_{g}(t) \delta v_{g}\left(t^{\prime}\right)}=\overline{v^{2}} f\left(t-t^{\prime}\right)
\end{aligned}
$$

The overline stands for the ensemble average, the characteristic function $f\left(t-t^{\prime}\right)$ will be specified below.

The starting point of our investigation is the canonical transformation which converts the gate potential into a time-dependent operator involving one of the generators of the group characterizing the dynamical symmetry of a complex QD 8,9,14 This transformation applied to the Schroedinger operator $-i \hbar(\partial / \partial t)+H_{d o t}$ gives $8,12,13$

$$
\widetilde{H}_{d o t}=U_{1}^{-1} H_{d o t} U_{1}-i \hbar U_{1}^{-1} \frac{\partial U_{1}}{\partial t},
$$

with

$$
U_{1}=\exp \left[i \phi_{1}(t) n_{1}\right]
$$

and the phase $\phi_{1}(t)$ given by

$$
\phi_{1}(t)=\frac{1}{\hbar} \int^{t} d t^{\prime} v_{g}\left(t^{\prime}\right)
$$

One may apply the Hausdorff expansion to the first term in 2.8

$$
\widetilde{H}_{d o t}(t)=H_{d o t}^{(0)}+\sum_{m} \frac{(i)^{m}}{m !}\left[S_{1},\left[S_{1} \ldots\left[S_{1}, H_{d o t}^{(0)}\right]\right] \ldots\right]
$$


where $S_{1}=\phi_{1}(t) n_{1}$, and $H_{d o t}^{(0)}$ includes all timeindependent terms from 2.3

It is expedient to introduce "even" and "odd" hopping operators

$$
T_{1 j}^{( \pm)}=\sum_{\sigma}\left[d_{1 \sigma}^{\dagger} d_{j \sigma} \pm d_{j \sigma}^{\dagger} d_{1 \sigma}\right]
$$

To the lowest orders in $V_{g}$, the time dependent part of the dot the Hamiltonian acquires the form

$$
\delta H_{\mathrm{dot}}(t)=-V \sum_{j}\left(i \tilde{\phi}_{1}(t) T_{1 j}^{(-)}+\frac{1}{2} \overline{\phi_{1}(t)^{2}} T_{1 j}^{(+)}\right)
$$

where

$$
\begin{aligned}
& \tilde{\phi}_{1}(t)=\frac{1}{\hbar} \int^{t} d t^{\prime} \tilde{v}_{g}\left(t^{\prime}\right) \\
& \overline{\phi_{1}(t)^{2}}=\frac{\overline{v^{2}}}{\hbar^{2}} \int^{t} d t^{\prime} \int^{t} d t^{\prime \prime} f\left(t^{\prime}-t^{\prime \prime}\right) .
\end{aligned}
$$

Thus, we obtain the effective time-dependent dot Hamiltonian

$$
\widetilde{H}_{\mathrm{dot}}=H_{\mathrm{dot}}^{(0)}+\delta H_{\mathrm{coh}}(t)+\delta H_{\mathrm{stoch}}(t),
$$

where the time-dependent perturbation contains a coherent component [the first term in (2.13)] and a stochastic one [the second term in (2.13)]. In many cases the coherent perturbation may be easily taken into account in an adiabatic approximation, $, 8,12$ whereas the second term in (2.15) results in the stochastization of the quantum state of the complex QD.

We are interested in the influence of such a timedependent perturbation on the electron cotunneling through complex QDs in Kondo regime. To investigate this influence one should derive the effective spin Hamiltonian from 2.1 by means of a time-dependent Schrieffer-Wolff (SW) transformation 8,12 taking into account the perturbation $\delta H_{\text {dot }}(t)$ in (2.15). The adiabatic component of this perturbation results in temporal oscillations of Kondo transparency and enhances the tunnel conductance on average,$\frac{15}{,}$ whereas the stochastic component of the gate potential is detrimental for Kondo tunneling. It results in the loss of coherence of the Kondo singlet state and in the smearing of zero bias anomaly in tunnel conductance. In accordance with the general approach to decoherence phenomena, $\underline{16}$ one should discriminate between the decoherence of the ground state of a quantum-mechanical ensemble and its manifestation at a finite energy/temperature. In the latter case one should argue in terms of dephasing due to elastic and inelastic scattering. Both processes are relevant in our system (see I).

In the two next sections we will show how the coherent part of $\delta H(t)$ results in the conversion of the charge input signal to a Kondo response, and why its stochastic component brings an end to this process.

\section{DOUBLE QUANTUM DOT IN T-SHAPE GEOMETRY. EVEN ELECTRON OCCUPATION}

We start with the T-shaped DQD (Fig. 19) and consider the case of even occupation $\mathcal{N}=2$ with one electron per potential well. In this two-terminal geometry the irreducible set $c_{\beta k \sigma}^{\dagger}$ consists of two combinations of source $(s)$ and drain $(d)$ leads. Only even standing wave $c_{e k \sigma}=\left(c_{s k \sigma}+c_{d k \sigma}\right) / \sqrt{2}$ enters $H_{\text {tun }}$ (2.5), and we omit in what follows the index $\beta=e$. The intradot indices $j j^{\prime}$ have two values 1,2 . We consider a DQD with two equivalent wells $\left(Q_{j}=Q\right)$ and assume that the timeindependent component $V_{g}(0)$ of the gate voltage modifies the single-electron spectrum in such a way that the charge transfer excitation due to hopping $H_{12}^{0}$ (2.4) is a relatively soft excitation with the energy $\Delta_{12} \ll Q$ (see Fig. 2a.)

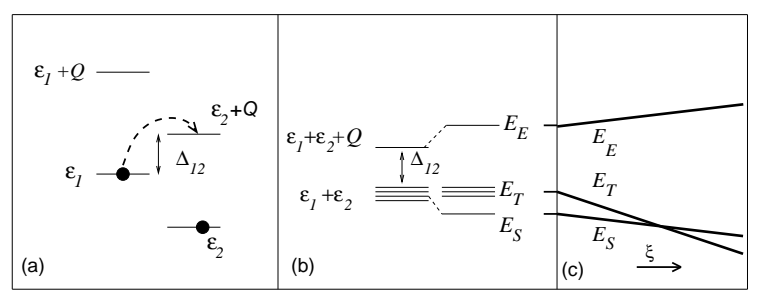

FIG. 2: Energy levels of an isolated DQD. (a) Single electron levels. The interdot tunneling $V$ is shown by a dashed arrow; (b) Two-electron levels $E_{\Lambda}$; (c) Evolution of $E_{\Lambda}$ with the growing of the scaling parameter $\xi=\ln D_{0} / D$ (see text for further details).

Under these conditions the low-lying part of the energy spectrum of DQD in the charge sector $\mathcal{N}=2$ consists of two singlet states and one triplet state: $\underline{14}$

$$
\begin{aligned}
& E_{S}=\varepsilon_{1}+\varepsilon_{2}-2 \alpha V, \\
& E_{T}=\varepsilon_{1}+\varepsilon_{2}, \\
& E_{E}=2 \varepsilon_{2}+Q_{2}+2 \alpha V .
\end{aligned}
$$

Here $\alpha=V / \Delta_{E S}$ is the effective indirect exchange parameter, which favors antiparallel orientation of electron spins in two valleys of the DQD. The ground state of the isolated DQD is the spin singlet $E_{S}$.

In spite of this, Kondo tunneling through DQD in Tshape geometry is possible under certain conditions, because the contact with lead electrons renormalizes the effective exchange in such a way that the singlet/triplet level crossing is possible. This result was obtained in Ref. 14 by means of a renormalization group (RG) technique 17 with scaling parameter $\xi=\ln D_{0} / D$, where $D_{0}$ and $D$ is the initial and current energy scales for electrons in the leads, respectively.

Having in mind this structure of low-lying states, it is convenient to represent $H_{d o t}$ in terms of Hubbard operators $X^{\Lambda \Lambda^{\prime}}=|\Lambda\rangle\left\langle\Lambda^{\prime}\right|$, where $|\Lambda\rangle$ are the eigenvectors 
corresponding to the eigenstates $E_{\Lambda}$ (3.1):

$$
\begin{aligned}
& H_{\mathrm{dot}}=\sum_{\Lambda} E_{\Lambda} X^{\Lambda \Lambda} \\
& \Lambda=T \nu, S, E, \quad \nu= \pm 1,0
\end{aligned}
$$

It is important for further calculations that the system of operators describing transitions between the levels of any multiplet consisting of two singlets and one triplet forms a closed set of generators of the $S O(5)$ algebra. Ten generators forming this algebra are packed into three vectors $\mathbf{S}, \mathbf{P}, \mathbf{M}$ and one scalar $A$. Vectors describe transitions within the triplet $T \nu$, between the triplet and the singlet $S$ and between the triplet and the singlet $E$, respectively. The scalar $A$ stands for transitions between the singlets $S$ and $E$. All these operators may be expressed via Hubbard operators $X^{\Lambda \Lambda^{\prime}}$ (see Refs. 8, 9,10 for further details). One may rewrite the Hamiltonian $H_{\text {dot }}$ in terms of these generators

$$
H_{\mathrm{dot}}=\frac{1}{2}\left(E_{T} \mathbf{S}^{2}+E_{S} \mathbf{P}^{2}+E_{E} \mathbf{M}^{2}\right)+Q(\hat{\mathcal{N}}-2)^{2}
$$

Besides, the hopping operator $T_{12}^{(-)}$(2.12) may be represented as

$$
T_{12}^{(-)}=i A \sqrt{2}
$$

Finally, the Casimir operator $\mathcal{C}$ for the $S O(5)$ group is

$$
\mathcal{C}=\mathbf{S}^{2}+\mathbf{P}^{2}+\mathbf{M}^{2}+A^{2}=4 .
$$

Using Eqs. (3.3), (3.4) as input data in (2.11) and (2.13), we obtain the effective Hamiltonian (2.15) in the following form (see I)

$$
H_{\mathrm{dot}}(t)=\frac{1}{2}\left(E_{T} \mathbf{S}^{2}+\tilde{E}_{S}(t) \mathbf{P}^{2}+\tilde{E}_{E}(t) \mathbf{M}^{2}\right)-\mu(\mathcal{C}-4)
$$

with

$$
\begin{aligned}
& \tilde{E}_{S}(t)=E_{S}-\delta_{\mathrm{coh}}(t)-\delta_{\text {stoch }, S}(t) \\
& \tilde{E}_{E}(t)=E_{E}+\delta_{\mathrm{coh}}(t)+\delta_{\text {stoch }, E}(t)
\end{aligned}
$$

Here coherent and stochastic corrections enter in the form of time-dependent "energy levels" :

$$
\begin{aligned}
\delta_{\text {coh }}(t) & =\left(2 V^{2} / \Delta_{E S}\right) \phi_{1}^{2}(t), \\
\delta_{\text {stoch }, S}(t) & =\left(V^{2} / 4 \Delta_{S T}\right) \overline{\phi_{1}^{2}(t)}, \\
\delta_{\text {stoch }, E}(t) & =\left(V^{2} / 4 \Delta_{E T}\right) \overline{\phi_{1}^{2}(t)},
\end{aligned}
$$

$\Delta_{\Lambda \Lambda^{\prime}}=\left|E_{\Lambda}-E_{\Lambda^{\prime}}\right|$. As was pointed out in Ref. 8, a charge perturbation cannot directly affect spin degrees of freedom, and the triplet level $E_{T}$ remains time independent.

It is seen from (3.7), that the coherent component results in the time-dependent shift of energy levels. It may be treated as an adiabatic correction provided the timedependent perturbation is weak enough, $\delta_{\text {coh }}(t) \ll \Delta_{E T}$. Below we adopt this adiabatic approximation.
Unlike the coherent renormalization, stochastic components are not "in phase", i.e., $\delta_{\text {stoch }, S}(t) \neq \delta_{\text {stoch, } E}(t)$. This inequality makes the constraint imposed on spin dynamics by the Casimir operator $\mathcal{C}$ (3.5) "fragile" for the $S O(5)$ group. Another source of stochasticity is the last term in Eq. (2.8). In lowest order in the stochastic correlation functions (2.7) its contribution is $\sim \hbar n_{1} \overline{\dot{\phi}_{1}(t) \phi_{1}(t)}$. This contribution may be converted into additional 4-th order corrections to Eqs. (3.7), and we neglect it in the following calculations.

A time-dependent SW transformation of the Hamiltonian (2.1) is performed by means of canonical transformation $\tilde{H}=U_{2} H U_{2}^{-1}$. The phase $\Upsilon$ in the matrix $U_{2}=\exp i \Upsilon$ is given by

$$
\Upsilon(t)=\sum_{k \sigma}\left[v_{k}^{S}(t) X^{S r_{\bar{\sigma}}} c_{k \sigma}+v_{k}^{E}(t) X^{E r_{\bar{\sigma}}} c_{k \sigma}-H . c .\right],
$$

The coefficients $v_{k}^{S}(t), v_{k}^{E}(t)$ are fixed through the condition

$$
H_{\text {tun }}+\left[\Upsilon,\left(H_{\text {dot }}+H_{\text {band }}\right)\right]=i \hbar \frac{\partial \Upsilon}{\partial t} .
$$

The solution of this equation is described in I, and the resulting cotunneling Hamiltonian has the form

$$
H_{\text {cotun }}(t)=J_{0}^{T} \mathbf{S} \cdot \mathbf{s}+J^{S}(t) \mathbf{P} \cdot \mathbf{s}+J^{E}(t) \mathbf{M} \cdot \$ 3
$$

Again, pure spin scattering is not affected by charge perturbation, but time-dependent spin-flip transitions in the leads described by the two last terms in 3.11 arise due to the fact that the dynamical symmetry of the dot spin multiplet is activated by the time-dependent gate potential. Like in Eq. (3.6), the time-dependent coupling parameters in the SW Hamiltonian contain both coherent and stochastic components,

$$
J^{\Lambda}(t)=J_{0}^{\Lambda}+J_{\text {ad }}^{\Lambda}(t)+J_{\text {stoch }}^{\Lambda}(t)
$$

$(\Lambda=S, E)$. The time-dependent corrections to $J_{0}^{\Lambda}$ are calculated in Appendix A. Now we are well prepared to study the contribution of adiabatic and stochastic corrections to Kondo tunneling.

\section{A. Coherent input signal.}

We study here the transformation of the monochromatic gate potential

$$
\tilde{v}_{g}(t)=\tilde{v}_{g} \cos \Omega t
$$

into a coherent (adiabatic) Kondo response under the condition $\Omega \ll \Delta_{S T}$ and $\delta_{\text {coh }}(t) \ll \Delta_{E T}$. Then the phase $\widetilde{\phi}_{1}(t)$ has a simple form

$$
\widetilde{\phi}_{1}(t)=\frac{\widetilde{v}_{g}}{\hbar \Omega}(\sin \Omega t-1)
$$


We calculate the coherent (adiabatic) response at low enough energy and temperature, where the last term in (3.11) may be omitted. Then we remain in the reduced $\{S, T\}$ part of Hilbert states, i.e. assume that the Kondo temperature $T_{K} \sim \Delta_{S T}$ is valid. The dynamical symmetry of the reduced adiabatic (ad) effective Hamiltonian

$$
\begin{aligned}
H_{S W} & =H_{\mathrm{dot}}^{(\mathrm{ad})}+H_{\mathrm{cotun}}^{(\mathrm{ad})}, \\
H_{\mathrm{dot}} & =\frac{1}{2}\left(E_{T} \mathbf{S}^{2}+E_{S}(t) \mathbf{P}^{2}\right)+Q(\hat{\mathcal{N}}-2)^{2}, \\
H_{\mathrm{cotun}}^{(\mathrm{ad})} & =J_{0}^{T} \mathbf{S} \cdot \mathbf{s}+\left[J_{0}^{S}+J_{\mathrm{ad}}^{S}(t)\right] \mathbf{P} \cdot \mathbf{s}
\end{aligned}
$$

is $S O(4)$.

The adiabatic part of the time-dependent Hamiltonian may be incorporated in a Haldane-type RG theory, $\underline{8,12,17}$ As a result, the levels $E_{\Lambda}$ acquire self energies scaled with the parameter $\xi=\ln \left(D_{0} / D\right)$, namely $M_{\Lambda}=\alpha_{\Lambda} \xi$ so that

$$
\begin{aligned}
E_{T} & \rightarrow E_{T}-\alpha_{T} \xi, \\
E_{S}(t) & \rightarrow E_{S}-\alpha_{S}(t) \xi,
\end{aligned}
$$

The self energy $M_{S}$ depends parametrically on time. The coefficients $\alpha_{S, T}$ were calculated in Ref. 14, and it was shown there that the inequality $\alpha_{T}>\alpha_{S}$ is always valid due to existence of excited singlet level $E_{E}$. Due to this inequality, the S/T level crossing may occur at this stage of renormalization (see Fig. 2k), so that the ground state of the system is triplet to the right of the crossing point. The time dependent factor $\alpha_{S}(t)$ describes parametrically slow variations of the scaling trajectory due to adiabatic (coherent) corrections given by Eqs. (3.7) and (3.8).

The expression for $J_{\mathrm{ad}}^{S}(t)$ derived in Appendix A reads explicitly (see Eq. A3):

$$
J_{\mathrm{ad}}^{S} \approx \frac{\sqrt{2} W^{2}}{\epsilon_{2}-M_{S}} \widetilde{\phi}_{1}(t)
$$

(one may neglect the time-dependence of the denominator).

It is known from Kondo theory for quantum dots with $S O(4)$ symmetry $\underset{14,20,21}{ }$ that the Kondo temperature is a very sharp function of $\Delta_{S T}$ with a maximum $T_{K 0}$ at $\Delta_{S T}=0$ (see Fig. 3, left column). It has an intermediate asymptotic behaviour for positive $\Delta_{S T}$ where the ground state of DQD is a triplet

$$
\frac{T_{K}(t)}{T_{K 0}}=\left[\frac{T_{K 0}}{\Delta_{S T}(t)}\right]^{\eta},
$$

valid in an intermediate asymptotic regime for positive $\Delta_{S T}$ at $T_{K 0} / \Delta_{S T} \lesssim 1$. Here $\eta<1$ is a universal constant. This sharp dependence is a key to the chargespin transformation mechanism, which is especially effective in the vicinity of the triplet/singlet critical point $\Delta_{S T}=0$.

Next, we estimate the influence of the trembling signal on the tunnel conductance $G(T, t)$ at given $T>T_{K}$ in

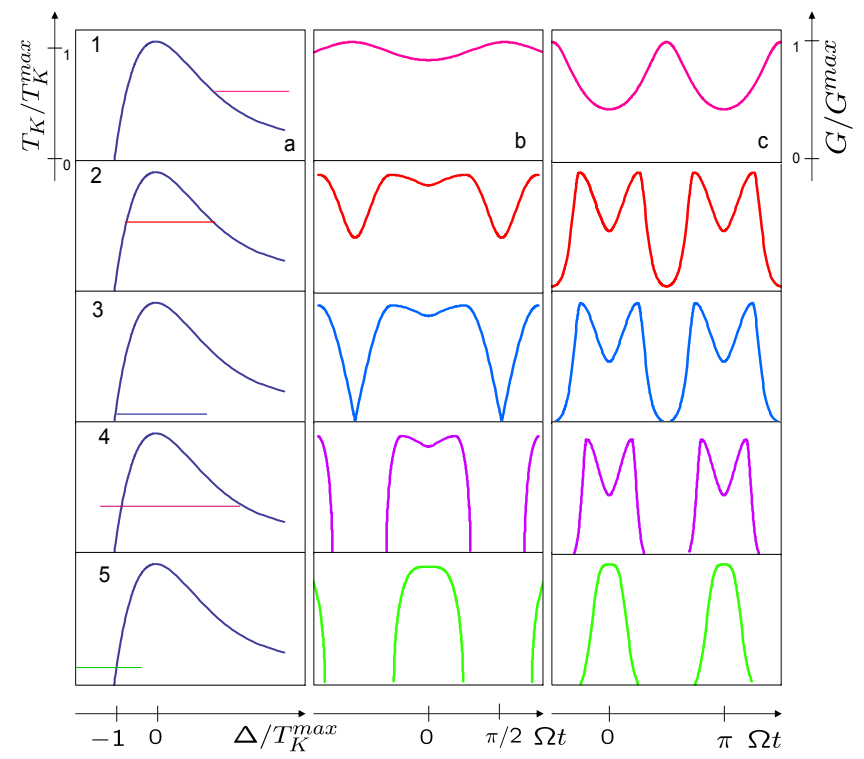

FIG. 3: (Color online) Left column: $T_{K}$ as a function of $\Delta_{S T}$. Middle column: time dependent $T_{K}(t)$ corresponding to the evolution of $T_{K}\left(\Delta_{S T}\right)$ in the left column. The intervals over which the evolution is followed are shown by straight lines in the left column. Right column: Evolution of ZBA in the conductance according to Eq. (3.19). See the text for further discussion.

a situation where the temporal variations of $\Delta_{S T}(t)=$ $E_{S}-E_{T}-\delta_{\text {coh }}(t)$ changes $T_{K}(t)$ close to the above mentioned degeneracy point $\Delta_{S T}=0$. In this hightemperature weak coupling regime the zero bias anomaly (ZBA) in tunnel conductance obeys the law

$$
\frac{G}{G_{0}} \sim \ln ^{-2}\left(T / T_{K}\right)
$$

Substituting (3.18) in (3.19), one gets

$$
\frac{G(t)}{G_{0}} \sim\left(\operatorname { l n } \left(T / T_{K 0}-\eta \ln \left(T_{K 0} / \Delta_{S T}(t)\right)^{-2}\right.\right.
$$

The results of the numerical analysis of the Kondo response $T_{K}(t)$ to the periodical input given by Eq. (3.13) as well as the adiabatically varying conductance (3.19) are presented in Fig. 3 It is seen from this analysis that the input sinusoidal potential $\tilde{v}_{g} \cos \Omega t$ transforms into periodic oscillations of the Kondo-related ZBA in $G(t)$. The sinusoid is reproduced with a slight distortion when the gap $\Delta_{S T}(t)$ remains positive under temporal perturbation (row 1 in Fig. 3). Additional minima impart the "Kremlin wall" shape to the periodic curve $G(t)$ when the sign of $\Delta_{S T}(t)$ changes under the periodic perturbation (rows 2,3). The same regime with larger amplitude $\tilde{v}_{g}$ may result in complete suppression of Kondo tunneling due to the periodical triplet-singlet crossover (row 4). Finally, if the system remains completely in the singlet sector $\Delta_{S T}(t)<0$ near the crossover point, the charge perturbation results in a pulsed Kondo output signal (row 5). 
It is worth noticing that this mechanism of adiabatic transformation of a charge signal into a Kondo response is close to that proposed for Kondo shuttling ${ }^{15}$ where the source of time-dependence are the nanoelectromechanical oscillations of a quantum dot with even occupation between two leads.

The reference Kondo temperature $T_{K 0}$ is given by the equation

$$
T_{K 0}=\bar{D} e^{-\frac{1}{\left(j_{0}^{T}+j^{S}\right)}}
$$

where $j^{\Lambda}=\rho_{F} J^{\Lambda}, \rho_{F}$ is the density of electron states in the leads at the Fermi level, and $\bar{D}$ is the characteristic scale of these states in SW regime. ${ }^{14}$ This temperature also oscillates adiabatically in time due to the correction (3.17) to the second term in the exponent. One may estimate adiabatic oscillations $\delta T_{K 0}^{\text {ad }}$ in the lowest order in $j_{\mathrm{ad}}^{S} /\left(J_{0}^{T}+J_{0}^{S}\right) \ll 1$. One derives from (3.21)

$$
\delta T_{K 0}^{\mathrm{ad}}(t) \approx T_{K 0} \frac{j_{\mathrm{ad}}^{S}(t)}{\left(j_{0}^{T}+j_{0}^{S}\right)^{2}} \sim T_{K 0} \frac{\widetilde{\phi}_{1}(t)}{j_{0}^{T}} .
$$

These temporal oscillations only weakly distort the curves shown in Fig. 3 because the Kondo temperature in these curves changes by the order of its magnitude due to oscillations changing the sign of $\Delta_{S T}(t)$. One may roughly estimate this effect by averaging (3.22) over an oscillation period. Like in the Kondo shuttling, 15 this averaging results in an effective enhancement of $T_{K 0}$ which resembles the Debye-Waller enhancement of neutron scattering intensity because of a nonzero average quadratic displacement induced by phonon vibrations.

\section{B. Stochastic input}

We begin with the discussion of the influence of an incoherent input on the spin state of quantum dot isolated from a metallic reservoir. As is known from the general theory of dynamical symmetries, $\frac{9}{9}$ only those states from the total manifold are involved in its formation whose energies are comparable with the energy scale of the interaction which breaks the symmetry of the Hamiltonian. In our case this scale is determined by the Kondo temperature $T_{K} \sim \Delta_{S T} \ll \Delta_{E T}$.

\section{Fluctuations of the global constraint}

The mechanism of conversion of the stochastic component $\delta v_{g}(t)$ of the input signal into a stochastic spin response is quite unusual. Instead of dephasing due to time-dependent spin flip processes, $\frac{12}{2}$ stochastization of the energy spectrum of DQD results in the loss of a Curietype spin response at some characteristic energy $\zeta$. This effect is related to the time dependence of the factors $\delta_{\text {stoch, } \Lambda}(t)$ (3.8) in the Hamiltonian (3.6). Indeed, inserting (3.7) into (3.6), one may write the stochastic part of $H_{\text {dot }}$ in the form

$$
H_{\mathrm{dot}}^{\text {stoch }}=\left[\delta_{\text {stoch }, S}(t) \mathbf{P}^{2}-\delta_{\text {stoch }, E}(t) \mathbf{M}^{2}\right] / 2
$$

Unlike the adiabatic part of time dependent energies $E_{\Lambda}(t)$, this term describes spin fluctuations related to the dynamical symmetry of DQD. In the reduced singlet/triplet subspace the exact confinement preserved by the last term in (3.6) obeying $S O(5)$ dynamical symmetry transforms into fluctuating confinement in the effective Hamiltonian,

$$
\begin{aligned}
H_{d o t}(t) & =\frac{1}{2}\left(E_{T} \mathbf{S}^{2}+E_{S} \mathbf{P}^{2}\right) \\
& -\mu\left(\mathbf{S}^{2}+\mathbf{P}^{2}-3\right)+\delta_{\text {stoch }, S}(t) \mathbf{P}^{2}
\end{aligned}
$$

where the $S O(4)$ symmetry is preserved only approximately. Thus the stochastic component of the dot Hamiltonian given by the correlation functions (3.8) appears explicitly in the constraint.

It follows from 3.24 that this component survives even in the asymptotic regime, $T \ll \Delta_{S T}$, where the $\mathrm{T} / \mathrm{S}$ excitations are quenched in the Kondo scattering, but the singlet component of the spin multiplet still influences the constraint via its stochastic constituent. The effective dot Hamiltonian in this limit has the form

$$
H_{d o t}(t)=\frac{1}{2} E_{T} \mathbf{S}^{2}-\mu\left(\mathbf{S}^{2}-2\right)-\delta_{\text {stoch }, S}(t) \mathbf{S}^{2}
$$

so that the fluctuations of the charge S/E gap may be transformed into the fluctuations of the spin constraint. This unusual situation is considered below in greater detail.

To investigate the influence of stochastic corrections of $\mu$ on the spin properties of isolated dot, we rewrite the Hamiltonian (3.25) in a fermionized form

$$
H_{d o t}(t)=\sum_{\nu=0, \pm 1}\left[E_{T} / 2-\mu(t)\right] f_{\nu}^{\dagger} f_{\nu} .
$$

Here $f_{\nu}$ are spin fermions representing the $\mathrm{S}=1$ triplet,,$\frac{8,9}{9}$ and the time-dependent chemical potential for spin fermions is defined as $\mu(t)=\mu_{0}-\delta_{\text {stoch }, S}(t)$. The stochastic component of $\mu$ may be treated as a random potential in the time domain, which describes the fluctuations of global fermionic constraint $\underline{8}$ The problem of propagation of spin fermions in a random time-dependent potential may be considered by means of the "cross technique" 24 developed for the study of electron propagation in a field of impurities randomly distributed in real space.

In Ref. 8 the short-time (white-noise) fluctuations of a global fermionic constraint have been considered. The noise correlation in this limit is delta-like

$$
D\left(t-t^{\prime}\right)=\hbar^{2}\left\langle\mu(t) \mu\left(t^{\prime}\right)\right\rangle=r_{0} \delta\left(t-t^{\prime}\right) .
$$

Such a description presumes that the chemical potential suddenly "shaken" at any moment does not keep memory 
about its previous value (correlation time equals zero). The decoherence time calculated in Born approximation is given by

$$
\hbar / \tau_{d} \sim r_{0}
$$

Here we propose another realization of the stochastic potential, which corresponds to the situation when the chemical potential varies slowly in time $(\sim \exp (-\gamma t))$. A very long relaxation time $\tau_{r} \sim 1 / \gamma$ with small $\gamma$ is assumed, so that the noise correlation is given by

$$
D(\omega)=\lim _{\gamma \rightarrow 0} \frac{2 \zeta^{2} \gamma}{\omega^{2}+\gamma^{2}}=2 \pi \zeta^{2} \delta(\omega)
$$

In this limit the averaged spin propagator describes the ensemble of states with chemical potential $\mu=$ const in a given state, but this constant is random in each realization 25

The problem of decoherence of the spin state in a stochastically perturbed DQD in this limit can be mapped on the so-called Keldysh mode126,27,28 originally formulated for systems which are $\delta$-correlated in the momentum space impurity scattering potential. The problem can be solved exactly and the decoherence time is defined by the variance $\zeta^{2}$ of the Gaussian correlation (see below). We look for a solution of the time-dependent model where time is the only current coordinate in the system.

The spin-fermion propagator at $T=0$ is defined as

$$
G_{T \nu}^{R}\left(t-t^{\prime}\right)=\left\langle f_{\nu}(t) f_{\nu}^{\dagger}\left(t^{\prime}\right)\right\rangle_{R}=-i\left\langle\left[f_{\nu}(t) f_{\nu}^{\dagger}\left(t^{\prime}\right)\right]_{+}\right\rangle .
$$

We sum the perturbation series for the Fourier transform of this Green's function

$$
G^{R}(\varepsilon)=g(\varepsilon)\left[1+\sum_{n=1}^{\infty} A_{n} \zeta^{2 n} g^{2 n}(\varepsilon)\right]
$$

Here $g(\varepsilon)=(\varepsilon+i \delta)^{-1}$ is the free spin-fermion propagator with $E_{T} / 2-\mu_{0}=0$ taken as the reference energy. The index $\nu$ is omitted, since the fluctuations of $\mu$ are related to the global $U(1)$ symmetry. The noise correlation function (3.28) is normalized in such a way that corresponding vertices are dimensionless. The Feynman

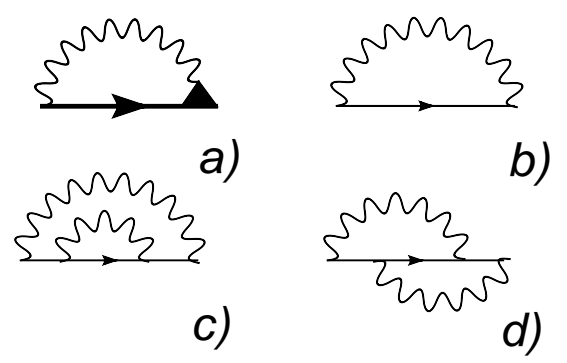

FIG. 4: (a) Feynman diagrams for the self energy with vertex corrections; (b) Bare self energy; (c) First line correction; (d) First vertex correction.

diagrams for the self-energy $\Sigma(\epsilon)$ are shown in Fig. 4.
The key features of the Keldysh model stem from the fact that all self energy diagrams in a given order are equivalent due to the delta-function character of the correlation function $D(\omega)$ (3.28). As a result, the contribution of all diagrams in a given order $n$ is completely determined by the combinatorial coefficient $A_{n}=(2 n-1)$ !! giving the total number of diagrams corresponding to all possible pairwise connections of $n$ vertices by wavy lines. Then the exact analytical equation for the self energy may be derived, $, 26,27,28$

$$
\Sigma(\epsilon)=\int \frac{d \omega}{2 \pi} \Gamma(\epsilon, \epsilon-\omega ; \omega) G(\epsilon-\omega) D(\omega)
$$

Here $\Gamma$ is the full vertex (triangle), $G$ is the full Green function (thick line) and $D$ is the noise correlation function (wavy line) in Fig. 4a. Evaluation of the integral (3.31) with the $\delta$-functional $D(\omega)$ (3.28) gives

$$
\Sigma(\epsilon)=\zeta^{2} \Gamma(\epsilon, \epsilon ; 0) G(\epsilon)
$$

In order to find $G(\epsilon)$ we use the Ward identity illustrated by Fig. 5n which connects the triangular vertex and the Green's function (GF)

$$
\Gamma(\epsilon, \epsilon ; 0)=\frac{d G^{-1}(\epsilon)}{d \epsilon}
$$

Then the Dyson equation for the spin-fermion propaga-
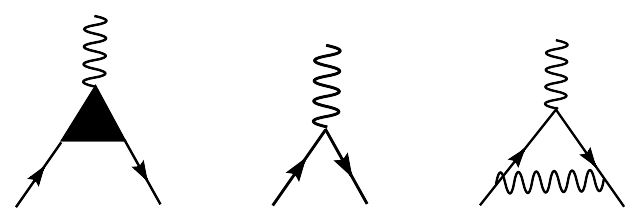

FIG. 5: Diagrams for the vertex $\Gamma$.

tor is transformed with the help of (3.33) into an ordinary differential equation:

$$
\zeta^{2} \frac{d G}{d \epsilon}+\epsilon G-1=0
$$

This equation is supplemented by the boundary condition 27,28

$$
G(\epsilon \rightarrow \infty)=\frac{1}{\epsilon}
$$

The solution of (3.34) satisfying the boundary condition (3.35) is given by

$$
G^{R}(\epsilon)=\frac{1}{\zeta \sqrt{2 \pi}} \int_{-\infty}^{\infty} e^{-z^{2} / 2 \zeta^{2}} \frac{d z}{\epsilon-z+i \delta}
$$

Remarkably, the spin-fermion GF in this model has no poles, singularities or branch cuts. The solution (3.36) represents the set of spin states under a stochastically fluctuating chemical potential averaged with a Gaussian exponent characterized by the variance $\zeta^{2}$. 
Let us investigate the spin response of this "stochasticized" DQD. To calculate the spin susceptibility, it is convenient to make an analytic continuation of $G^{R}$ on the imaginary semi-axes of complex energies, i.e. to go over to the thermodynamical Matsubara Green's functions:

$$
\mathcal{G}\left(i \epsilon_{n}\right)=\frac{1}{\zeta \sqrt{2 \pi}} \int_{-\infty}^{\infty} e^{-z^{2} / 2 \zeta^{2}} \frac{d z}{i \epsilon_{n}-z}
$$

The spin (triplet) susceptibility at finite temperatures defined by the diagrams of Fig. 6 may be calculated by means of these functions. The spin susceptibility with
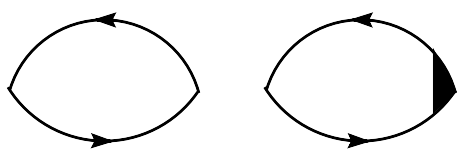

FIG. 6: Diagrams for bare and dressed spin susceptibility.

vertex corrections is determined as

$$
\begin{aligned}
& \chi\left(i \omega_{m}\right)= \\
& T \sum_{n} \mathcal{G}\left(i \omega_{m}+i \epsilon_{n}\right) \mathcal{G}\left(i \epsilon_{n}\right) \Gamma\left(i \epsilon_{n}, i \epsilon_{n}+i \omega_{m} ; i \omega_{m}\right)
\end{aligned}
$$

The Ward identity 3.33 provides us with the exact equation for the vertex function

$$
\Gamma^{R}(\epsilon, \epsilon ; 0)=\frac{\epsilon G^{R}-1}{\zeta^{2}\left(G^{R}\right)^{2}},
$$

giving access to the exact evaluation of the static susceptibility $\chi(0)$ (right diagram on Fig. 6). Combining (3.38) with (3.37) and (3.39), we find

$$
\chi(0)=\frac{1}{\sqrt{8 \pi} \zeta} \int_{-\infty}^{\infty} d y e^{-y^{2} / 2} y \tanh \left(\frac{y \zeta}{2 T}\right)
$$

The asymptotic behavior of the static susceptibility $\chi(0)$ is

$$
\chi(0)= \begin{cases}C_{\mathrm{C}} / T, & T \gg \zeta \\ C_{\mathrm{K}} / \zeta, & \zeta \gg T\end{cases}
$$

where $C_{\mathrm{C}}$ and $C_{\mathrm{K}}$ are constants. At high $T$ the behavior of the dot is Curie-like with Curie constant $C_{\mathrm{C}}$ modified by averaging. At low $T$ the noise dispersion $\zeta$ plays the role of an effective temperature in the Keldysh model with the corresponding constant $C_{\mathrm{K}}$ in the numerator.

There is a great simplification in the calculations of the dynamic susceptibility at temperatures $T \gg \zeta$. We notice that $\Gamma \rightarrow 1$ in this limit since transferred energy exceeds the dispersion of the noise spectrum. Under this condition one can neglect the vertex corrections, and the spin susceptibility is given by

$$
\chi\left(i \omega_{m}\right)=\frac{1}{2 \pi \zeta^{2}} \int_{-\infty}^{\infty} \frac{d z_{1} d z_{2} e^{-\frac{z_{1}^{2}+z_{2}^{2}}{2 \zeta^{2}}}}{\cosh \left(\frac{z_{1}}{2 T}\right) \cosh \left(\frac{z_{2}}{2 T}\right)} \frac{\sinh \left(\frac{z_{2}-z_{1}}{2 T}\right)}{i \omega_{m}+z_{2}-z_{1}}
$$

Performing the analytic continuation, one gets the following equation for the imaginary part of the spin response function at real frequencies:

$$
\begin{aligned}
& \operatorname{Im} \chi^{R}(\omega)= \\
& -\frac{e^{-\frac{\omega^{2}}{4 \zeta^{2}}}}{\zeta^{2}} \tanh \left(\frac{\omega}{2 T}\right) \int_{-\infty}^{\infty} d q \frac{e^{-\frac{q^{2}}{4 \zeta^{2}}}}{\frac{\cosh \left(\frac{q}{2 T}\right)}{\cosh \left(\frac{\omega}{2 T}\right)}+1} .
\end{aligned}
$$

Thus $\operatorname{Im} \chi^{R} \sim \omega$ at small $\omega \ll T$.

The real part of the static susceptibility is given by

$$
\begin{aligned}
& \operatorname{Re\chi }^{R}(\omega)= \\
& \frac{1}{2 \pi \zeta^{2}} \int_{-\infty}^{\infty} d u \frac{u \sinh \left(\frac{u}{2 T}\right)}{u^{2}-\omega^{2}} e^{-\frac{u^{2}}{4 \zeta^{2}}} \times \\
& \times \int_{-\infty}^{\infty} d q \frac{e^{-\frac{q^{2}}{4 \zeta^{2}}}}{\cosh \left(\frac{u}{2 T}\right)+\cosh \left(\frac{q}{2 T}\right)}
\end{aligned}
$$

where the principal part of the integrals is taken.

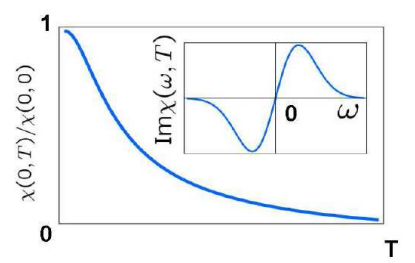

FIG. 7: (Color online) Static susceptibility $\chi(0, T)$. The inset shows a frequency dependence of $\operatorname{Im} \chi$ with a maximum at $\omega \sim \zeta$.

It follows from (3.40), 3.42 and 3.43 and from results of numerical calculations presented in Fig. 7 that the low-frequency response of a DQD in the Keldysh regime has nothing to do with the behavior of a free spin. This means that in spite of the fact that at high $T$ a DQD behaves like a quantum object with spin 1 , it looses at $\{\omega, T\} \ll \zeta$ the generic characteristics of a localized spin due stochastization, hence it cannot serve as a source of Kondo screening at low energies.
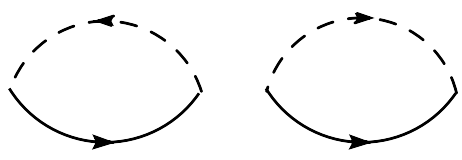

FIG. 8: Diagrams for spin-electron loops responsible for the Kondo effect. Here solid and dashed lines stand for spin-fermion propagators $\mathcal{G}\left(i \epsilon_{n}\right)$ and electron propagators $g\left(\mathbf{p}, i \omega_{n}\right)$, respectively.

A direct calculation of spin-electron loops responsible for Kondo screening (Fig. 8) confirms this conclusion. These second order perturbation theory corrections to the spin-electron vertex are given by

$$
\mathcal{T}^{(2)}\left(i \epsilon_{n}\right) / J \sim J T \sum_{m} \int \frac{d \mathbf{p}}{(2 \pi)^{3}} \mathcal{G}\left(\mathbf{p}, i \omega_{m}\right) \mathcal{G}\left(i \epsilon_{n}+i \omega_{m}\right)
$$


Substituting Green's functions into this integral, we have

$$
\mathcal{T}^{(2)}\left(i \epsilon_{n}\right) / J \sim(\rho J) \int_{-\infty}^{\infty} d z e^{-\frac{z^{2}}{2 \zeta^{2}}} \int_{T}^{D} d \xi \frac{\tanh \left(\frac{\xi}{2 T}\right)}{i \epsilon_{n}-\xi+z}
$$

This vertex correction reflects the averaging of the standard Kondo vertex with the Gaussian distribution of chemical potentials. Evaluating the integral, one obtains a combination of logarithmic, hypergeometric and imaginary error functions

$$
\begin{gathered}
\mathcal{T}^{(2)}(\epsilon \rightarrow 0) / J=\rho_{0} J \ln (\sqrt{2 C} D / \zeta)+ \\
+\frac{1}{2} \rho_{0} J\left[{ }_{1} F_{1}\left(\frac{3}{2}, 2, \frac{T^{2}}{2 \zeta^{2}}\right)\left(\frac{T}{\zeta}\right)^{2}-\pi \operatorname{Erfi}\left(\frac{T}{\sqrt{2} \zeta}\right)\right]
\end{gathered}
$$

where $\gamma=\ln C$ is the Euler constant. In two limiting cases of low and high temperatures relative to the dispersion $\zeta$ of noise spectrum, it leads to the following compact expressions

$$
\mathcal{T}^{(2)}(\epsilon \rightarrow 0) / J=\left\{\begin{array}{cc}
\rho J \ln (D / T), & T \gg \zeta \\
\rho J \ln (D / \zeta), & \zeta \gg T
\end{array}\right.
$$

We conclude from here that the variance $\zeta^{2}$ predetermines the energy/temperature cut-off (similarly to the Kondo-spin glass problem ${ }^{34}$ ). This result correlates with the above observation that at low $T \ll \zeta$ the magnetic excitations in stochasticized DQD loose the properties of spin flip processes which are essential for Kondo screening. The characteristic decoherence time is given by:

$$
\hbar / \tau_{d} \sim \zeta
$$

If $\zeta \ll T_{K}$, the noise effect is seen in the behavior of the magnetic susceptibility as a logarithmic correction,

$$
\chi(T)=\chi_{C}\left(1-\ln ^{-1}\left(\max (T, \zeta) / T_{K}\right)+\ldots\right),
$$

but to study the influence of $\delta v_{g}$ (2.6) on the Kondo processes at finite temperatures one should also consider the dephasing effects.

We comment also the S-T response function

$$
\chi_{P}^{\alpha \beta}\left(t-t^{\prime}\right)=-i\left\langle\left[P_{\alpha}(t), P_{\beta}\left(t^{\prime}\right)\right]\right\rangle \rightarrow \delta_{\alpha \beta} \chi_{P}\left(i \omega_{n}\right)
$$

which corresponds to the bare loop represented in Fig. 6a, where one of the two lines corresponds to the singlet fermionic GF while the other one represents the triplet fermionic GF. Since the singlet line is not affected by the noise, only one of the GFs in the loop suffers from Gaussian averaging.

The straightforward calculations lead to the following answer for the imaginary part

$$
\begin{aligned}
& \operatorname{Im} \chi_{P}^{R}(\omega) \sim \\
& -\frac{1}{\zeta} \frac{\sinh \left(\frac{\omega}{2 T}\right)}{\sinh \left(\frac{\Delta_{S T}}{2 T}\right)}\left[\frac{\exp \left(-\frac{\left(\Delta_{S T}+\omega\right)^{2}}{2 \zeta^{2}}\right)}{\cosh \left(\frac{\Delta_{S T}+\omega}{2 T}\right)}+\frac{\exp \left(-\frac{\left(\Delta_{S T}-\omega\right)^{2}}{2 \zeta^{2}}\right)}{\cosh \left(\frac{\Delta_{S T}-\omega}{2 T}\right)}\right]
\end{aligned}
$$

and the real part of the susceptibility

$$
\begin{aligned}
& \operatorname{Re\chi }_{P}^{R}(\omega) \sim \\
& \frac{1}{\zeta} \int_{-\infty}^{\infty} d z \frac{\left(z-\Delta_{S T}\right) \sinh \left(\left(z-\Delta_{S T}\right) / 2 T\right)}{\left(z-\Delta_{S T}\right)^{2}-\omega^{2}} \times \\
& \times \frac{e^{-\frac{z^{2}}{2 \zeta^{2}}}}{\cosh (z / 2 T) \cosh \left(\Delta_{S T} / 2 T\right)}
\end{aligned}
$$

The static susceptibility which mimics the Curie law at very large temperatures $T \gg\left(\Delta_{S T}, \zeta\right)$, is suppressed exponentially $\sim \exp \left(-\Delta_{S T} / T\right)$ at low temperatures $T \ll$ $\left(\Delta_{S T}, \zeta\right)$ and has an intermediate asymptotic behaviour $\chi_{P}(0) \sim 1 / \zeta \ln (\zeta / T)$ if $\Delta_{S T} \ll T \ll \zeta$ while $\chi_{P}(0) \sim$ $1 / \Delta_{S T}$ when $\zeta \ll T \ll \Delta_{S T}$. The real part of the dynamic susceptibility taken at the resonance frequency $\omega= \pm \Delta_{S T}$ grows as $1 / \zeta \ln (\zeta / T)$ when the temperature is lowered. The exponential suppression does not occur since $\Delta_{S T}$ is compensated by the external frequency. We therefore conclude that the noise may strongly affect the non-equilibrium electric-field-induced Kondo transport in the regime when the singlet is a ground state of DQD while the access to the triplet state is facilitated by the applied gate voltage.

\section{Fluctuations of the scattering phase}

In accordance with the general approach to decoherence and dephasing effects, $\frac{16}{r}$ the latter phenomena arise due to scattering processes at finite energy and/or temperature. These processes are described by the effective cotunneling Hamiltonian (3.11). The main contribution to dephasing is given by the term

$$
H_{\text {cotun }}^{(\text {stoch }}=J_{\text {stoch }}^{S}(t) \mathbf{P} \cdot \mathbf{s}
$$

Here $J_{\text {stoch }}^{S}(t)$ is the stochastic component of the indirect exchange integral calculated in Appendix A (Eq. A3). To reveal the dephasing mechanism, one should notice that the parameter $\overline{J_{\mathrm{st}}^{S}(t)}$

$$
\overline{J_{\mathrm{st}}^{S}(t)}=\frac{W^{2}}{\epsilon_{2}-M_{S}} \frac{2 V}{\Delta_{E S}}\left(\frac{\overline{v_{g}(t) \phi_{1}(t)}}{\epsilon_{2}}-\overline{\phi_{1}(t)^{2}}\right)
$$

is in fact the modification of the effective SW exchange due to temporal fluctuations of the intradot exchange $2 \alpha V$, which is nothing but the gap $\Delta_{S T}$ (see Eq. 3.1). Unlike the similar term $\delta_{\text {stoch }}(t)$ in Eqs. (3.7), (3.24), it does not influence the modulus of the vector $\mathbf{P}$, but the components of this vector, and thereby it affects the components of the spin vector $\mathbf{S}$ via scattering processes illustrated by Fig. 9 The time-dependent exchange vertex (3.49) is taken in the form $J_{s o} \varphi_{s}(t)$, so that the fluctuating part in parenthesis is represented by a single mode $\varphi(t)$.

The wavy lines in the diagram stand for the correlation functions $S\left(t-t^{\prime}\right)=\left\langle\varphi(t) \varphi\left(t^{\prime}\right)\right\rangle$, the solid line in 

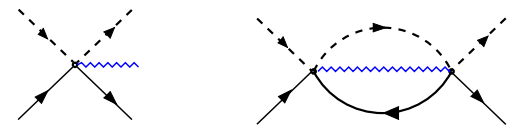

FIG. 9: Left: bare vertex in the fluctuation part of the exchange Hamiltonian (3.48). Right: first non-adiabatic correction to the exchange vertex $J_{0}^{T}$ in (3.11).

the vertex correction corresponds to the bare retarded propagator $g_{s}\left(t-t^{\prime}\right)=\left\langle f_{s}(t) f_{s}^{\dagger}\left(t^{\prime}\right)\right\rangle_{R}$ for pseudofermion excitations representing the singlet mode in the $S O(5)$ multiplet 9.14 As was noted in I, the eventual source of dephasing is the gauge fluctuation field induced by nonadiabatic excitations in the non-diagonal operator $\mathbf{P}$ with components $P_{\nu}=f_{s}^{\dagger} f_{\nu}$.

The problem of dephasing due to slow fluctuations $S(\omega \rightarrow 0)$ was analyzed in I, so we do not repeat here the corresponding calculations. The net result of this analysis is that the dephasing processes are relevant at high enough temperatures, and at $T \ll \Delta_{S T}$ dephasing is effectively quenched.

\section{TRIPLE QUANTUM DOT IN A TRIANGULAR GEOMETRY. ODD ELECTRON OCCUPATION}

In this section we sort out the coherent and stochastic components of the weak probe time-dependent potential $v_{g}(t)(2.6)$ applied to the triangular TQD shown in Fig. 10. To make the mathematical treatment more transparent, we consider the three-terminal geometry, so that the system possesses a perfect triangular symmetry $C_{3 V}$. It is convenient to enumerate both the dots and the leads by the numbers $1,2,3$. The bias is supposed to be created between the leads 2,3 , and the role of a passive terminal 1 is to serve as a reservoir for Kondo screening of the electron spin in the dot. The gate voltage $v_{g}(t)$ is applied to one of the electrodes forming the dot 1 .

Then the band Hamiltonian has the form

$$
H_{\text {band }}=\sum_{j=1,2,3} \varepsilon_{j k} c_{j k \sigma}^{\dagger} c_{j k \sigma},
$$

Correspondingly, the tunnel Hamiltonian is written as

$$
H_{t u n}=W \sum_{j k \sigma}\left(c_{j k \sigma}^{\dagger} d_{j \sigma}+H . c .\right)
$$

We study the excitation spectrum of the TQD in the charge sector $\mathcal{N}=1$. The spin degrees of freedom obey the $S U(2)$ symmetry, and all dynamical symmetry effects are related to orbital degrees of freedom. We will show here how the influence of charge input on the orbital degrees of freedom may be converted into Kondo response.

The spectrum of the TQD was discussed in Refs. 10,22.
This dot is described by the Hamiltonian

$$
\begin{aligned}
& H_{d o t}^{(0)}=\epsilon \sum_{j=1}^{3} \sum_{\sigma} d_{j \sigma}^{\dagger} d_{j \sigma}+Q \sum_{j} n_{j \uparrow} n_{j \downarrow} \\
& +Q^{\prime} \sum_{\langle j l\rangle} \sum_{\sigma} n_{j \sigma} n_{l \sigma^{\prime}}+V \sum_{\langle j l\rangle} \sum_{\sigma}\left(d_{j \sigma}^{\dagger} d_{l \sigma}+H . c .\right) .
\end{aligned}
$$

Here $n_{j \sigma}=d_{j \sigma}^{\dagger} d_{j \sigma},\langle j l\rangle=\langle 12\rangle,\langle 23\rangle,\langle 31\rangle, Q$ and $Q^{\prime}$ are intradot and interdot charging energies $\left(Q \gg Q^{\prime}\right), V$ is the interdot tunneling amplitude. In the case $\mathcal{N}=1$ charging terms are irrelevant and (4.3) is easily diagonalized

$$
H_{d o t}^{(0)}=\sum_{\Gamma, \sigma} \varepsilon_{\Gamma} d_{\Gamma \sigma}^{\dagger} d_{\Gamma \sigma}
$$

Here the index $\Gamma=A, E_{ \pm}$stands for irreducible representations of the symmetry group of equilateral triangle. The basis of this representation is given by the eigenfunctions

$$
\begin{aligned}
& d_{A, \sigma}^{\dagger}=\left(d_{1 \sigma}^{\dagger}+d_{2 \sigma}^{\dagger}+d_{3 \sigma}^{\dagger}\right) / \sqrt{3}, \\
& d_{E_{ \pm}, \sigma}^{\dagger}=\left(d_{1 \sigma}^{\dagger}+e^{ \pm 2 i \varphi} d_{2 \sigma}^{\dagger}+e^{ \pm i \varphi} d_{3 \sigma}^{\dagger}\right) / \sqrt{3} ;
\end{aligned}
$$

( $\varphi=2 \pi / 3$ ) The ground state is the orbital singlet $A$, and the orbital degrees are quenched at low temperatures $T \ll V$.

The triangular geometry provides us with a new possibility for controlling quantum tunneling ${ }^{10}$. The tunnel current may be driven by means of an external magnetic field oriented normally to the plane of triangle, because the electron spectrum of electrons in the TQD is a function of the magnetic flux $\Phi$ through the triangle in such a geometry. As a result orbital degrees of freedom may be activated at finite magnetic field, and the possibility opens to realize a charge-spin conversion mechanism already in the case of the TQD occupied by a single electron $(\mathcal{N}=1)$. The electron energy spectrum at finite $\Phi$ is

$$
\varepsilon_{\Gamma}(\Phi)=\epsilon-2 V \cos \left(p-\frac{\Phi}{3}\right) .
$$

and the values of $p=0,2 \pi / 3,4 \pi / 3$ correspond respectively to $\Gamma=A, E_{+}, E_{-}$. At zero $\Phi$ the states $\varepsilon_{E \pm}$ form degenerate doublet excitations. At finite $\Phi$ the electron acquires chirality, the doublet is split, the levels evolve in accordance with (4.6) and at $\Phi_{n}=(n+1 / 2) \Phi_{0}$ the ground state becomes doubly degenerate (here $\Phi_{0}$ is a quantum of magnetic flux, $n= \pm 1, \pm 2, \ldots)$. The level evolution is shown in Fig. 10 a. The dynamical symmetry $S U(4)$ is involved in the signal transformation at $\Phi$ close to $\Phi_{n}$, or in other words, at $\varepsilon_{\Gamma}-\varepsilon_{\Gamma^{\prime}} \sim T_{K}$ for any pair of states $\Gamma, \Gamma^{\prime}$. Figure 10 a shows the evolution of the energy levels in the interval $0 \leqslant \Phi \leqslant 3 \Phi_{0}$, but the real periodicity of the spectrum is of course $\Phi_{0}$ because at finite magnetic field all three states may be converted into each other by the appropriate choice of the gauge. 

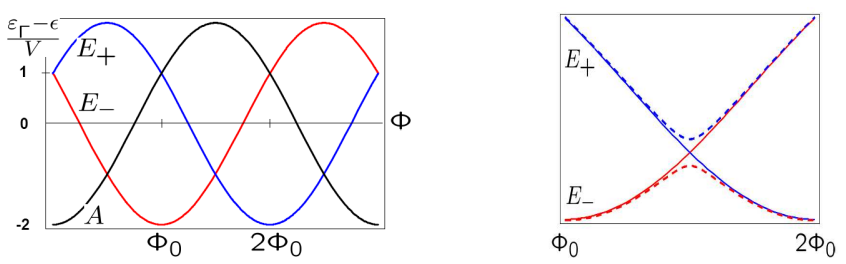

FIG. 10: (Color online) Left: Evolution of energy levels of a TQD in a magnetic field. Right: Zoomed level crossing around $\Phi=3 \Phi_{0} / 2$.

To make the notation symmetric we will consider below the sector $\Phi_{0} \leqslant \Phi \leqslant 2 \Phi_{0}$ shown in Figure [10(b). In this sector two states $E_{ \pm}$become nearly degenerate at $\Phi$ approaching $3 \Phi_{0} / 2$. When the symmetry of TQD is perfect, the point $\Phi=3 \Phi_{0} / 2$ is a point of accidental degeneracy, in which the symmetry of TQD is $S U(4) \stackrel{10}{\Perp}$. This symmetry is broken by the potential $v_{g}(t)$. As a result level crossing transforms into anticrossing (dashed lines in Fig. 10, left panel).

In order to calculate the contribution of $\delta H_{\text {dot }}(t)$ in the charge-spin conversion we need the matrix elements of the operators $T_{1 j}^{( \pm)}(2.12)$ in the basis $|\Gamma\rangle$ of the $C_{3 v}$ point group, in particular those involved in the above level crossing/anticrossing in the sector $\Phi_{0} \leqslant \Phi \leqslant 2 \Phi_{0}$. The relevant matrix elements in the subspace $\left|E_{ \pm}\right\rangle$are

$$
\begin{aligned}
& \left\langle E_{ \pm}\left|H_{d o t}^{(1)}\right| E_{ \pm}\right\rangle=-2 V \sin \varphi \phi_{1}(t), \\
& \left\langle E_{ \pm}\left|H_{d o t}^{(1)}\right| E_{\mp}\right\rangle=0
\end{aligned}
$$

and

$$
\begin{aligned}
& \left\langle E_{ \pm}\left|H_{d o t}^{(2)}\right| E_{ \pm}\right\rangle=-V \cos \varphi \phi_{1}^{2}(t), \\
& \left\langle E_{ \pm}\left|H_{d o t}^{(2)}\right| E_{\mp}\right\rangle=-V e^{i \varphi} \phi_{1}^{2}(t)
\end{aligned}
$$

It follows from (4.7), that the first-order term gives a purely adiabatic contribution $\sim \tilde{\phi}_{1}(t)$ (2.14). Secondorder corrections (first line in Eq. 4.8) slightly change the adiabatic renormalization. The stochastic signal $\sim \overline{\phi_{1}^{2}(t)}$ arises from the off-diagonal matrix elements (second line in Eq. 4.8).

\section{A. Coherent input signal}

Following Ref. 10, we use the irreducible representations $\left\{A, E_{ \pm}\right\}$not only for dot eigenstates (4.5) but also for lead states

$$
\begin{aligned}
& c_{A, \sigma}^{\dagger}=\sum_{k}\left(c_{1 k \sigma}^{\dagger}+c_{2 k \sigma}^{\dagger}+c_{3 k \sigma}^{\dagger}\right) / \sqrt{3} \\
& c_{E_{ \pm}, \sigma}^{\dagger}=\sum_{k}\left(c_{1 k \sigma}^{\dagger}+e^{ \pm 2 i \varphi} c_{2 k \sigma}^{\dagger}+e^{ \pm i \varphi} d_{3 k \sigma}^{\dagger}\right) / \sqrt{3} .
\end{aligned}
$$

in the three-terminal geometry.

In zero magnetic field the ground state is degenerate, the singly occupied TQD works as an effective spin $1 / 2$, so that the effective spin Hamiltonian has the standard form $J \mathbf{S} \cdot \mathbf{s}_{A A}$, where only fully symmetric combinations with $\Gamma=A$ of lead and dot electrons are represented. Orbital degrees of freedom become relevant near the crossing points, so that the effective Kondo Hamiltonian has the form

$$
H_{\text {cotun }}=\sum_{\Gamma \Gamma^{\prime}} J_{\Gamma \Gamma^{\prime}} \vec{S}_{\Gamma \Gamma^{\prime}} \vec{s}_{\Gamma^{\prime} \Gamma}+J_{o} \overrightarrow{\mathcal{T}} \vec{\tau}
$$

in the representation (4.5), (4.9). The spin operator for lead electrons is determined as $\mathbf{s}_{\Gamma \Gamma^{\prime}}^{i}=$ $\sum_{\mathbf{k k}^{\prime}} c_{\Gamma, \mathbf{k} \sigma}^{\dagger} \hat{\tau}_{i} c_{\Gamma^{\prime}, \mathbf{k}^{\prime} \sigma^{\prime}}$. Due to the orbital degeneracy $\left(E_{+}=E_{-}\right)$, one more vector, namely the pseudospin vector $\overrightarrow{\mathcal{T}}$ defined as

$$
\begin{aligned}
\mathcal{T}^{+} & =\sum_{\sigma}\left|E_{+}, \sigma\right\rangle\left\langle E_{-}, \sigma\right|, \quad \mathcal{T}^{-}=\left[\mathcal{T}^{+}\right]^{\dagger} \\
\mathcal{T}^{z} & =\frac{1}{2} \sum_{\sigma}\left(\left|E_{+}, \sigma\right\rangle\left\langle E_{+}, \sigma|-| E_{-}, \sigma\right\rangle\left\langle E_{-}, \sigma\right|\right) .
\end{aligned}
$$

together with its counterpart for lead electrons is involved in Kondo tunneling. There five vectors provide 15 generators of the SU(4) group. The effective Kondo Hamiltonian consists of 6 terms with corresponding exchange vertices, three of these vertices are relevant (including that for pseudospin interaction), and the corresponding Kondo temperature $T_{K}^{(E)}$ for $\Delta_{ \pm}=0$ exceeds the zerofield Kondo temperature $T_{K}^{(A)}$ by a factor of five (see Ref. 10 for details). Similarly to the case of ST degeneracy, deviation from the level-crossing point results in a sharp decrease of $T_{K}$, although in this case the peak is symmetric relative to the zero gap point (Inset in the left panel of Fig. 11). The width of this peak may be estimated as $\sim T_{K}^{(E)}$.

In the vicinity of the level crossing point the $E_{ \pm}$orbital components of the spin operators in (4.10) are involved in Kondo screening, provided the difference $\Delta_{ \pm}=$ $\left|E_{+}-E_{-}\right| \sim T_{K}$. Like in the case of a DQD (3.18), the Kondo temperature itself depends on the level distance, $T_{K}=T_{K}\left(\Delta_{ \pm}\right)$. We work in the adiabatic regime and incorporate matrix elements (4.7), (4.8) in the energy terms $\varepsilon_{ \pm}(4.6)$. We conclude from these equations and from Fig. 10 that only the non-diagonal matrix elements $\left\langle E_{ \pm}\left|H_{d o t}^{(2)}\right| E_{\mp}\right\rangle$ are relevant. Mixing of two branches $\sim \phi_{1}^{2}$ results in a time-dependent lifting of degeneracy of the orbital doublet at zero magnetic field, $\Phi=0$, and at $\Phi \approx 3 \Phi_{0} / 2$. In agreement with the general rule for the Kondo effect in presence of dynamical symmetry 10,20,35, $T_{K}\left(\Delta_{ \pm}\right)$is maximum for $\Delta_{ \pm}=0$, but unlike the case of DQD (Fig. 3), the curve $T_{K}\left(\Delta_{ \pm}\right)$is symmetric around its maximum (see Fig. 11] inset to the right panel). Now we may repeat the procedure proposed for the DQD in the previous section and calculate the time-dependent response of the ZBA peak in tunnel conductance to a coherent input signal (4.8). Two types of adiabatic temporal oscillations $T_{K}(t)$ are presented in the left panel of Fig. 11. If $\Delta_{ \pm}$oscillates between zero and some maxima due 

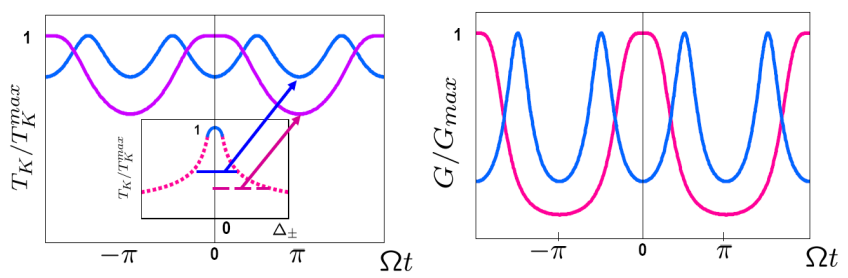

FIG. 11: (Color online) Left panel: Time dependent $T_{K}$ corresponding to the evolution of $\Delta_{ \pm}$in TQD. Inset: $T_{K}$ as a function of $\Delta_{ \pm}$. The intervals of this evolution are shown by straight lines. Right panel: Time dependent ZBA in the conductance in accordance with the evolution of $T_{K}$.

to fluctuations of the gate voltage, $T_{K}(t)$ reaches its maximum at $\Omega t=2 \pi n$. The tunnel conductance $G(t)$ shown in the right panel evolves with the same periodicity in accordance with Eq. (3.19). If $\Delta_{ \pm}$oscillates symmetrically around zero value, the period is halved, and $G(t)$ reaches minima at $\Omega t=\pi n$. In the general case when $\Delta_{ \pm}$varies between $-\Delta_{a}$ and $+\Delta_{b}$, the oscillations of $G(t)$ are periodic but not monochromatic. Experimentally one may turn from one regime to another by changing the magnetic field (shifting the value of $\Phi$ ) in the vicinity of the point $\Phi=3 \Phi_{0} / 2$.

We see that the situation with the $S U(2) \rightarrow S U(4) \rightarrow$ $S U(2)$ crossover is close to the case of the $S O(4) \rightarrow$ $S O(5) \rightarrow U(1)$ crossover discussed in the previous section from the point of view of the conversion of the charge signal into Kondo response. Due to the fact that the orbital degrees of freedom are involved in the formation of an effective exchange, the perturbation $v_{g}(t)$ directly affects Kondo tunneling by means of a time-dependent lowering of the point symmetry of the triangle induced by the gate voltage.

\section{B. Incoherent input signal}

The stochastization of orbital degrees of freedom in a TQD is induced by the term $\delta H_{d o t}^{(\text {stoch) }}(t)$ (2.15), (4.8). The relevant part is

$$
\Delta^{(\text {stoch })}(t)=\left\langle E_{-}\left|H_{\operatorname{dot}}^{\text {stoch }}\right| E_{+}\right\rangle=-V \overline{\phi_{1}^{2}(t)} e^{i \varphi}
$$

These stochastic inter-level transitions are responsible for the fluctuation induced avoided crossing $E_{+}-E_{-}$(dashed lines in the right panel of Fig. 10). One may write the corresponding part of the Hamiltonian in terms of pseudospin operator $\overrightarrow{\mathcal{T}}$, as

$$
H_{\mathrm{dot}}^{\text {stoch }}=\Delta_{ \pm}^{(\text {stoch })}(t) \mathcal{T}^{-}+\Delta_{ \pm}^{*(\text { stoch })}(t) \mathcal{T}^{+}
$$

This term should be added to the effective Kondo Hamiltonian of TQD (4.10).

Thus in this case the TQD stochastic fluctuations of gate voltage induce random pseudospin scattering, and the Keldysh approximation (3.28) may be used for this type of random potential. However, unlike the DQD case, the scattering has a vector character, so that the fermionized Hamiltonian (4.13) has the form

$$
H_{\mathrm{dot}}^{\mathrm{stoch}}(t)=\varrho(t) g_{\downarrow}^{\dagger} g_{\uparrow}+\varrho^{*}(t) g_{\uparrow}^{\dagger} g_{\downarrow}
$$

where $\varrho(t)$ is a random scattering potential which stems from (4.13), $g_{\uparrow}$ and $g_{\downarrow}$ are "pseudospin-fermions" for vector $\overrightarrow{\mathcal{T}}$. Only the transversal component of pseudospin scattering is involved in the stochastic perturbation.

Then, following the pattern of the scalar model (3.28), we introduce the correlation function $C\left(t-t^{\prime}\right)=$ $\left\langle\varrho(t) \varrho^{*}\left(t^{\prime}\right)\right\rangle$ and its Fourier transform $\sim$ Gaussian variance $\xi$,

$$
C(\omega)=\lim _{\gamma \rightarrow 0} \frac{4 \xi^{2} \gamma}{\omega^{2}+\gamma^{2}}=4 \pi \xi^{2} \delta(\omega)
$$

The expansion of the Fourier transform of the Green's function for pseudospin operators

$$
F_{\sigma}^{R}\left(t-t^{\prime}\right)=\left\langle g_{\sigma}(t) g_{\sigma}^{\dagger}\left(t^{\prime}\right)\right\rangle_{R}
$$

[cf. Eq. (3.29)] has the same form as in the scalar Keldysh model, 26,28 , namely,

$$
F(\varepsilon)=f(\varepsilon)+\sum_{n=1}^{\infty} B_{n}(\sqrt{2} \xi)^{2 n} f^{2 n+1}(\varepsilon) .
$$

Here $f(\epsilon)=(\epsilon+i \delta)^{-1}$ is the free spin-fermion propagator, $B_{n}$ is the total number of $2 \mathrm{n}$-th order diagrams. The indices $R, \sigma$ are omitted here and below for the sake of brevity. The main advantage of the Keldysh model, namely the equivalence of all diagrams corresponding to various combinations of noise correlation functions in the self energy (Fig. 4) is still available. However, the essential part of the diagrams in $\Sigma(\epsilon)$ disappears due to the kinematic constraint $\mathcal{T}^{+} \mathcal{T}^{+}=0$ (or $g_{\sigma}^{\dagger} g_{\sigma}^{\dagger}=0$ ). This means that only the diagrams with the pseudospin operators ordered as ... $\mathcal{T}^{+} \mathcal{T}^{-} \mathcal{T}^{+} \mathcal{T}^{-} \ldots$ survive in the self energy (Fig. 4a).

A similar version of the cross technique in a real space arises in electron systems in the domain of longrange Gaussian fluctuations near the charge density wave (CDW) instability, although the physical mechanism is radically different (alternating incoming and outgoing Umklapp fluctuations of CDW order parameter in $1 \mathrm{D}^{28,29}$ and $2 \mathrm{D}^{30,31}$ systems) 32

One may represent the diagrams for the vector Keldysh model in the following way. The vertices on the fermion line have two colors (say, black and white) corresponding to the first and second term in the time-dependent Hamiltonian (4.14). The black and white vertices alternate and the wavy lines connect only the vertices of different colors. Therefore the perturbation series includes only the even order terms with equal number of black and white vertices. Following these rules the vertex correction presented by the 4 -th order diagram of Fig. $4 \mathrm{~d}$ disappears. One of nonzero 6-th order diagrams is shown 
in Fig. 12 (left). This is the first non-vanishing vertex correction to the self energy of the vector Keldysh model. The difference between scalar and vector Keldysh model
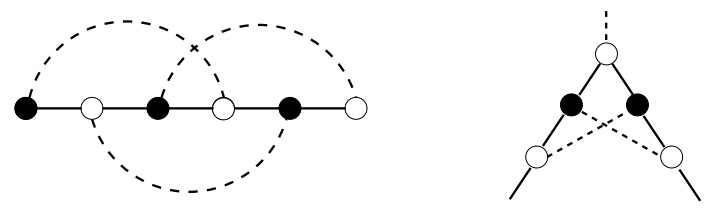

FIG. 12: First non-vanishing diagrams for the vertex (right) and the vertex correction to the fermionic self energy (left) in the vector Keldysh model. Black and white sites correspond to the two terms in the Hamiltonian (4.14); pseudospin correlation functions (4.16) are represented by dashed lines. is in this combinatorial coefficient. In the scalar model the coefficient $A_{n}=(2 n-1) ! !$, and summation of the perturbation series results in Eq. (3.36). In the vector model the coefficient $B_{n}=n$ ! due to above kinematic selection rules, and summation gives

$$
F(\epsilon)=\frac{1}{2} \int_{-\infty}^{+\infty} \frac{d x e^{-x^{2} / 2 \zeta^{2}}}{\zeta \sqrt{2 \pi}} \int_{-\infty}^{+\infty} \frac{d y e^{-y^{2} / 2 \zeta^{2}}}{\zeta \sqrt{2 \pi}}\left[\frac{1}{\epsilon-\sqrt{x^{2}+y^{2}}+i \delta}+\frac{1}{\epsilon+\sqrt{x^{2}+y^{2}+i \delta}}\right]
$$

(see Appendix B for the derivation of this result). We see that Eq. (4.18) is the natural generalization of the one-dimensional Gaussian averaging (3.36) characteristic for the scalar Keldysh model to the twodimensional Gaussian averaging of a vector random field with purely transversal $x y$ fluctuations. Only the modulus of the random field $r=\sqrt{x^{2}+y^{2}}$ is averaged with the Rayleigh distribution function $\mathcal{P}_{R}(\epsilon)=$ $\left(\epsilon / \zeta^{2}\right) \exp \left(-\epsilon^{2} / 2 \zeta^{2}\right)$, whereas the angular variable remains irrelevant due to the in-plane isotropy of the system. Like in the scalar model, the averaged pseudospinfermion Green's function has no singularities.

In order to find the Ward identity for the vertex and the differential equation for the Green's function generalizing Eqs. (3.33), (3.34) we propose for the vector Keldysh model the procedure which is an alternative to that used in ${ }^{27}$ for the scalar model. We calculate explicitly the derivative $d G_{\sigma}^{R}(\epsilon) / d \epsilon$ using the same method of summation of the series in the r.h.s. of Eq. (4.17) as the one which was used for the calculation of the integral representation (4.18) for the Green's function. This calculation (see Appendix B) gives the following result:

$$
\xi^{2} \frac{d F(\varepsilon)}{d \epsilon}=1-\epsilon F(\epsilon)\left(1-\frac{\xi^{2}}{\epsilon^{2}}\right)
$$

which is obviously the generalization of Eq. (3.34) with the similar boundary condition $F(\epsilon \rightarrow \infty)=\epsilon^{-1}$. One may rewrite Eqs. (3.34) and (4.19) in a unified way:

$$
\begin{aligned}
& \epsilon G(\epsilon)-1=\zeta^{2} G^{2}(\epsilon) \frac{d}{d \epsilon} G^{-1}(\epsilon) \\
& \epsilon F(\epsilon)-1=\xi^{2} F^{2}(\epsilon)\left\{\frac{1}{\epsilon} \frac{d}{d \epsilon}\left[\epsilon F^{-1}(\epsilon)\right]\right\}
\end{aligned}
$$

One may check straightforwardly that the functions $G(\epsilon)$ and $F(\epsilon)$, given by Eqs. (3.36) and (B2) are indeed solutions of differential equations (4.20). Then, appealing to Eq. (3.33), we define the vertex $\Gamma(\epsilon, \epsilon, 0)$ for the vector model as

$$
\Gamma=\frac{1}{\epsilon} \frac{d}{d \epsilon}\left(\epsilon F^{-1}(\epsilon)\right) .
$$

It is worth noting that the differential operator in the $\mathrm{r}$. h. s. of Eq. (4.21) is nothing but $\operatorname{div}_{\epsilon}$ in polar coordinates. This form reflects effective two-dimensionality of Gaussian averaging in the vector Keldysh model, which has been noticed already in Eq. (4.18). Equations (4.19) and (4.21) facilitate the calculation of response functions of TQD.

The stochastization of pseudospin manifests itself in the transformation of the response function $\chi_{\perp}(t)=$ $\left\langle\mathcal{T}^{-}(t) \mathcal{T}^{+}(0)\right\rangle_{R}$ shown in Fig. 6] Now the solid lines correspond to pseudofermion propagators $F_{\uparrow}(t)$ and $F_{\downarrow}(-t)$, and the vertex corrections are presented by the diagram 12 (right panel) and the higher-order diagrams of that sort. In order to calculate the pseudospin susceptibility at finite temperature we address to the equation

$$
\begin{aligned}
& \chi\left(i \omega_{m}\right)= \\
& T \sum_{n} \mathcal{F}_{\uparrow}\left(i \omega_{m}+i \epsilon_{n}\right) \mathcal{F}_{\downarrow}\left(i \epsilon_{n}\right) \Gamma\left(i \epsilon_{n}, i \epsilon_{n}+i \omega_{m} ; i \omega_{m}\right)
\end{aligned}
$$

similar to Eq. (3.38). Here $\mathcal{F}_{\sigma}\left(i \epsilon_{n}\right)$ is the Matsubaratype analytical continuation of the Fourier transform of the Green's function (4.16). Using the definition (4.21) of the vertex $\Gamma$ and the second equation from (4.20), we express the vertex as

$$
\Gamma\left(\epsilon_{n}, \epsilon_{n}, 0\right)=\frac{i \epsilon_{n} \mathcal{F}\left(i \epsilon_{n}\right)-1}{\xi^{2} \mathcal{F}^{2}\left(i \epsilon_{n}\right)}
$$


[cf. Eq. (3.39)]. Following the procedure which led to Eqs. 3.40 and 3.41 in scalar model, we obtain for $\chi_{\perp}(0)$ and its asymptotics the following equations

$$
\chi_{\perp}(0)=\frac{1}{\xi} \int_{0}^{\infty} y^{2} d y e^{-y^{2} / 2} \tanh \left(\frac{y \xi}{2 T}\right)
$$

and

$$
\chi(0) \sim\left\{\begin{array}{cc}
1 / T, & T \gg \xi \\
1 / \xi, & \xi \gg T
\end{array}\right.
$$

Thus the pseudospin in the vector model looses its local characteristics in the same way as the spin in the scalar model. Accordingly, stochastization affects the Kondo tunneling. To estimate this effect we calculated the electron-pseudofermion loops similar to those shown in Fig. 8, but with solid lines standing for $\mathcal{F}_{\sigma}\left(i \epsilon_{n}\right)$. Since the weak coupling approach works only in the limit $T, \xi \gg \Delta_{ \pm}$, the pseudo-gap in the spectrum does not affect the logarithmic behaviour of the Kondo-loop. The stochastization-induced level repulsion in the vector model excludes states within the pseudogap both when $T \gg \xi$ and $T \ll \xi$. In the latter case the infra-red Kondo cutoff is of the order of $\xi$. Therefore, the resonance Kondo tunneling in this case is controlled by spin degrees of freedom only in accordance with the $S U(2)$ Kondo effect paradigm.

We conclude from the above results, that the case of $S U(4)$ symmetry supported by the interplay between spin and orbital degrees of freedom in a TQD differs radically from the case of $S O(4)$ symmetry involving only the spin variables. In the latter case the external charge noise results in the stochastization of spin degrees of freedom, so that the DQD "looses" its spin moment at low enough energy/temperature. In the former case the spin $1 / 2$ is robust, and only the orbital (pseudospin) degrees of freedom are affected by the charge noise. Pseudospin stochastization means that the logarithmic divergences in the corresponding Kondo loops given by the diagrams similar to those of Fig. 8 are subject to a cut-off similar to that given by Eq. (3.41). As a result, only the spinelectron loops determine the Kondo screening at low $T$. Thus a noise induced $S U(4) \rightarrow S U(2)$ crossover takes place in TQD.

\section{CONCLUDING REMARKS}

In this paper we have demonstrated that the charge-to spin conversion of a time dependent input signal applied to the gate attached to a side well of a complex quantum dot is possible through several mechanisms involving the dynamical symmetry of this nano-object. Such a possibility arises when the multiplet involved in the dynamics of low-energy excitations includes both charge and spin degrees of freedom. In the case of $S O(5)$ symmetry characterizing the T-shaped DQD with even occupation, charge transfer singlet excitons are activated by $v_{g}(t)$, and the spin degrees of freedom are excited via the Casimir constraint. In the case of $S U(4)$ symmetry which determines the dynamics of TQD with single electron occupation, the signal $v_{g}(t)$ affects orbital (pseudospin) degrees of freedom, which are involved in the Kondo screening together with conventional spin states.

Both coherent and stochastic components of $v_{g}(t)$ are subject to a charge-spin transformation. The Kondo response to a coherent charge signal is close in its nature to oscillations of the Kondo temperature discussed in a context of Kondo shuttling. 15 The noise component introduces the stochastization of spin degrees of freedom. This stochastization may be complete in DQD, provided the variance of the Gaussian noise exceeds $T_{K}$. Then the low-energy cutoff results in the smearing and even the elimination of the Kondo-related ZBA in tunneling conductance. In TQD with $S U(4)$ symmetry only the charge (orbital) degrees of freedom are stochasticized. Strong enough noise may result in peculiar noise-induced quantum crossover $S U(4) \rightarrow S U(2)$, which may be controlled experimentally by varying the noise level in the input signal.

\section{APPENDIX A}

The time-dependent SW transformation may be performed in the adiabatic approximation. 12 In our case it gives the following expressions for the coupling constants controlling $T / S$ and $T / E$ transitions: ${ }^{8}$

$$
\begin{aligned}
J^{S}(t) & =\frac{W-w_{2}^{S}(t)}{\epsilon_{2}-M_{S}(t)} \cdot \frac{W\left(1-V / \Delta_{E S}(t)\right) \sqrt{2} \phi_{1}(t)}{\Delta_{E S}(t)}, \quad(\mathrm{A} 1) \\
J^{E}(t) & =\frac{W-w_{2}^{E}(t)}{\epsilon_{2}+Q_{2}+M_{E}(t)} \cdot \frac{W\left(1-V / \Delta_{E S}(t)\right) \sqrt{2} \phi_{1}(t)}{\Delta_{E S}(t)},
\end{aligned}
$$

with

$$
\begin{gathered}
M_{S}(t)=M_{S}-C_{S} \phi_{1}^{2}(t), \quad M_{E}(t)=M_{E}+C_{S} \phi_{1}^{2}(t) \\
w_{2}^{S}(t)=\frac{V W \sqrt{2} \phi_{2}^{S}(t)}{\Delta_{E S}}, \quad w_{2}^{E}(t)=\frac{V W \sqrt{2} \phi_{2}^{E}(t)}{\Delta_{E S}}, \\
\phi_{2}^{S}(t)=\phi_{1}(t)-\kappa_{2}^{S}(t), \quad \phi_{2}^{E}(t)=\phi_{1}(t)-\kappa_{2}^{E}(t), \\
\kappa_{2}^{S}(t)=v_{g}(t) / \epsilon_{2}, \quad \kappa_{2}^{E}(t)=v_{g}(t) /\left(\epsilon_{2}+Q_{2}\right) .
\end{gathered}
$$

To second order in $v_{g}(t)$ Eqs. A1 and A2 lead to the averaged time-dependent corrections to the coupling coefficients

$$
\begin{aligned}
& \overline{J^{S}(t)}=J_{\mathrm{ad}}^{S}+J_{\text {stoch }}^{S} \\
& \approx \frac{\sqrt{2} W^{2}}{\epsilon_{2}-M_{S}}\left(\tilde{\phi}_{1}(t)+\frac{\sqrt{2} V}{\Delta_{E S}}\left(\frac{\overline{v_{g}(t) \phi_{1}(t)}}{\epsilon_{1}}-\overline{\phi_{1}(t)^{2}}\right)\right)
\end{aligned}
$$


and a similar expression for $\overline{J^{E}(t)}$ where $\epsilon_{2}-M_{S}$ is replaced by $\epsilon_{2}+Q_{2}+M_{E}$.

\section{APPENDIX B}

To derive the pseudospin-fermion GF for the vector Keldysh model, we generalize the original Keldysh summation procedure 26,28 to the perturbation series (4.17). Using the integral representation for the $\Gamma$-function, $n !=$ $\int_{0}^{\infty} d z z^{n} e^{-z}$, we transform the series (4.17) into

$$
\left.F(\epsilon)=f(\epsilon)\left\{1+2 \sum_{n} \int_{0}^{\infty} t d t[t \xi f(\epsilon)]^{2 n}\right] e^{-t^{2}}\right\}
$$

(cf. Ref. 29). Here we substituted $t^{2}$ for the variable $z$. Then changing the order of summation and integration, we transform $F_{\sigma}(\epsilon)$ into the integral

$$
F(\epsilon)=\int_{0}^{\infty} 2 t d t \frac{f(\epsilon)}{1-2 t^{2} \xi^{2} f^{2}(\epsilon)} e^{-t^{2}}
$$

Taking into account the explicit form of the free pseudospin-fermion propagator, $f \epsilon)=(\epsilon+i \delta)^{-1}$, we change the integration variable once more, $t=u / \sqrt{2} \xi$ and put it into the form (B1)

$$
F(\epsilon)=\int_{0}^{\infty} \frac{u d u}{2 \xi^{2}}\left(\frac{1}{\epsilon-u+i \delta}+\frac{1}{\epsilon+u+i \delta}\right) e^{-u^{2} / 2 \xi^{2}}
$$

Next we introduce the "cartesian" coordinates, $x=$ $u \cos \phi, y=\sin \phi$, so that $u=\sqrt{x^{2}+y^{2}}$ and $d x d y=$ $u d u d \phi$. The angle-independent integral (B2) may be rewritten as

$$
\begin{aligned}
& F(\epsilon)=\frac{1}{4 \pi \xi} \int_{-\infty}^{+\infty} d x \int_{-\infty}^{+\infty} d y e^{-\left(x^{2}+y^{2}\right) / 2 \xi^{2}} \\
& {\left[\frac{1}{\epsilon-\sqrt{x^{2}+y^{2}}+i \delta}+\frac{1}{\epsilon+\sqrt{x^{2}+y^{2}}+i \delta}\right]}
\end{aligned}
$$

which is in fact the expression in Eq. (4.18).

In order to calculate the derivative $d F_{\sigma}^{R} / d \varepsilon$, we start with the same expansion (4.17). The analog of Eq. (B1) for the derivative has the form

$$
\frac{d F(\varepsilon)}{d \varepsilon}=-f^{2}(\varepsilon)\left[1+\int_{0}^{\infty} 2 t d t \frac{2\left(2 t^{2}-1\right) t^{2} \xi^{2} f^{2}(\varepsilon)}{1-2 t^{2} \xi^{2} f^{2}(\varepsilon)} e^{-t^{2}}\right]
$$

The subsequent variable change which gave Eq. (B2) for the GF gives for its derivative the following equation

$$
\frac{d F(\varepsilon)}{d \varepsilon}=-f^{2}(\varepsilon)\left[1+\frac{1}{2}\left(\frac{J_{4}}{\xi^{4}}-\frac{J_{2}}{\xi^{2}}\right)\right]
$$

where

$$
J_{n}=\int_{0}^{\infty} d z z^{n} \exp \left(-\frac{z^{2}}{2 \xi^{2}}\right)[f(\varepsilon-z)-f(\varepsilon+z)]
$$

After some manipulations, these integrals are represented via the $\mathrm{GF}$ for the vector model (B2):

$$
J_{2}=2 \varepsilon \xi^{2} F-2 \xi^{2}, \quad J_{4}=-4 \xi^{4}+\varepsilon^{2} J_{2}
$$

Substituting these integrals in Eq. (B4), we come after some algebra to the differential equation (4.19).
1 M.I. Dyakonov and V.I. Perel, Sov. Phys. JETP Lett. 13, 467 (1971).

2 S. Murakami, N. Nagaosa and S.-C. Zhang, Science 301, 1348 (2003); N. Sugimoto, S. Onoda, S. Murakami, and N. Nagaosa, Phys. Rev. B 73, 113305 (2006).

3 M.I. Dyakonov, Phys. Rev. Lett. 99, 126601 (2007).

${ }^{4}$ H.-F.Lü and Y. Guo, Appl. Phys. Lett. 91, 092128 (2007)

5 C. Caccamo, G. Pizzimenti, and M.P. Tosi, Nuovo Cimento B31, 53 (1976).

6 I. D'Amico and G. Vignale, Phys. Rev. B 62, 4853 (2000).

7 S. Li, T.F. George, X. Sun, and L.-S. Chen, J. Phys. Chem. B 111, 6099 (2007).

8 M.N. Kiselev, K. Kikoin, Y. Avishai, and J. Richert, Phys. Rev. B 74, 115306 (2006)

${ }^{9}$ K. Kikoin, Y. Avishai and M.N. Kiselev, Dynamical symmetries in nanophysics, in "Nanophysics, Nanoclusters and Nanodevices," (Nova Publishers, New York, 2006) pp. 3986; arXiv:cond-mat/0407063

10 T. Kuzmenko, K. Kikoin and Y. Avishai, Phys. Rev. Lett. 96, 046601 (2006).

11 A. Amato, T. Hatano, T. Kubo, Y. Tokura, D.G. Austing, and S. Tarucha. Physica E 40, 1322 (2008).

12 A. Kaminski, Yu. V. Nazarov and L.I. Glazman, Phys.
Rev. B 62, 8154 (2000).

13 C. Bruder and H. Schoeller, Phys. Rev. Lett. 72, 1076 (1994).

14 K. Kikoin and Y. Avishai, Phys. Rev. Lett. 86, 2090 (2001); Phys. Rev. B 65, 115329 (2002).

15 M.N. Kiselev, K. Kikoin, R.I. Shekhter, and V.M. Vinokur, Phys. Rev. B 74, 233403 (2006).

16 H.D. Zeh in Decoherence and the Appearance of a Classical World in Quantum Theory, eds. E. Joos et al (SpringerVerlag, Berlin, 2003) 2-nd ed., Chapter 9.

17 F.D.M. Haldane, Phys. Rev. Lett. 40, 416 (1978).

18 P.W. Anderson, J. Phys. C 3, 2436 (1970).

19 A. Kogan, G. Granger, M.A. Kastner, and D. GoldhaberGordon, H. Shtrikman, Phys. Rev. B 67, 113309 (2003).

20 M. Eto and Yu.V. Nazarov, Phys. Rev. Lett. 85, 1306 (2000), Phys. Rev. B 64, 085322 (2001); M. Eto, J. Phys. Soc. Jpn, 74, 95 (2005).

21 M. Pustilnik and L.I. Glazman, Phys. Rev. Lett. 85, 2993 (2000), Phys. Rev. B 64, 045328 (2001).

22 F. Delgado, Y.-P. Shim, M. Korkusinski, P. Hawrylak, Phys. Rev. B 76, 115332 (2007)

23 M.N. Kiselev, Int. J. of Mod. Phys. B, 20, 381 (2006).

24 S.F. Edwards, Phil. Mag. 3, 33, 1020 (1958). 
25 Similar problem of stochastization of the Landau-Zener (LZ) level crossing problem in two limiting cases (3.27) and (3.28) was considered by Y. Kayanuma, J. Phys. Soc. Jpn. 53, 108 (1984); 54, 2037 (1985), detailed theory of slow-noise-assisted LZ problem is given in Ho Ngoc Phien and M.N.Kiselev (to be published).

${ }^{26}$ L.V. Keldysh, "Semiconductors in strong electric field", D. Sci. thesis, Lebedev Institute, Moscow, 1965.

27 A.L. Efros, Sov. Phys.-JETP 32, 479 (1971) [Zh. Eksp. Teor. Fiz. 59, 880 (1970)].

28 M.V. Sadovskii, Diagrammatics (World Scientific, Singapore, 2005).

29 M.V. Sadovskii, Zh. Eksp. Teor. Fiz. 66, 1720 (1974) [Sov. Phys. - JETP 39, 845 (1974)].

${ }^{30}$ L. Bartosch and P. Kopietz, Eur. Phys. J. B 17, 555 (2000).
31 E.Z. Kuchinskii and M.V. Sadovskii, JETP 94, 654 (2002).

32 General formulation of Keldysh model in time domain with both scalar and vector fluctuations taken into account, was developed for the toy model of ensemble of two-level systems in Ref. M.N. Kiselev and K. Kikoin, JETP Letters 89, 114 (2009) [Pis'ma v ZhETF, 89, 133 (2009) ].

33 The derivation procedure is outlined in Appendix B in concern to a generalized (vector) Keldysh model.

34 M.N. Kiselev and R. Oppermann, JETP Lett 71, 250 (2000) [Pis'ma v ZhETF 71, 359 (2000)]

35 Y. Avishai and K. Kikoin, Tunneling through Quantum Dots with Discrete Symmetries, in "Encyclopedia of Complexity" (Springer Verlag, Berlin, 2008) ; arXiv:0801.3095 